\title{
A macroscopic model to reproduce self-organization at bottlenecks
}

\author{
Boris Andreianov* \\ Institut Denis Poisson, CNRS UMR 7013, Université de Tours, Université d'Orléans \\ Parc de Grandmont, 37200 Tours cedex, France \\ Abraham Sylla ${ }^{\dagger}$ \\ Institut Denis Poisson, CNRS UMR 7013, Université de Tours, Université d'Orléans \\ Parc de Grandmont, 37200 Tours cedex, France
}

\begin{abstract}
We propose a model for self-organized traffic flow at bottlenecks that consists of a scalar conservation law with a nonlocal constraint on the flux. The constraint is a function of an organization marker which evolves through an ODE depending on the upstream traffic density and its variations. We prove well-posedness for the problem, construct and analyze a finite volume scheme, perform numerical simulations and discuss the model and related perspectives.
\end{abstract}

\section{Introduction}

The LWR framework is the simplest one that can be used to describe macroscopically pedestrian/road traffic in a corridor or on a road. It takes the form

$$
\partial_{t} \rho+\partial_{x}(f(\rho))=0 .
$$

Above, $\rho=\rho(x, t) \in[0, R]$ is the density of pedestrians/cars at $(x, t)$. We assume that the flux function $f:[0, R] \rightarrow \mathbb{R}$ is Lipschitz continuous and bell-shaped, which are commonly used assumptions in traffic dynamics:

$$
f(\rho) \geq 0, f(0)=f(R)=0, \exists ! \bar{\rho} \in(0, R), f^{\prime}(\rho)(\bar{\rho}-\rho)>0 \text { for a.e. } \rho \in(0, R) .
$$

Point constraints were introduced in $[17,16]$ in the LWR model in order to account for localized in space phenomena that may occur at exits - such as traffic lights or tollgates in the context of road traffic - and which act as obstacles. To do so, one can impose a localized constraint on the flux such as

$$
\left.f(\rho)\right|_{x=0} \leq q(t) .
$$

One of the typical features of both vehicle and pedestrian flows is self-organization, see $[22,18,13]$ for empirical data that put in evidence this phenomenon. Here, we focus on self-organization near exits. We do not intend to model the different mechanisms behind self-organization, but only to reproduce its phenomenology. In [3] the authors attempted to reproduce self-organization with a model based on the LWR-flux constraint framework:

$$
\left\{\begin{array}{lr}
\partial_{t} \rho+\partial_{x}(f(\rho))=0 & \mathbb{R} \times(0, T) \\
\rho(x, 0)=\rho_{0}(x) & x \in \mathbb{R} \\
\left.f(\rho)\right|_{x=0} \leq p\left(\int_{\mathbb{R}} \rho(x, t) \mu(x) \mathrm{d} x\right) & t \in(0, T) .
\end{array}\right.
$$

\footnotetext{
*Boris.Andreianov@lmpt.univ-tours.fr
}

${ }^{\dagger}$ Abraham.Sylla@lmpt.univ-tours.fr 
Above, $\mu \in \mathbf{W}^{1,1}(\mathbb{R}) \cap \mathbf{C}^{1}(\mathbb{R})$ is a weight function, supported in a compact neighborhood upstream the exit, used to average the density around the exit and the nonincreasing Lipschitz function $p:[0, R] \rightarrow \mathbb{R}^{+}$models the exit efficiency. This kind of problems has been tremendously studied in the last decades $[16,6,14,4,19]$. In particular, the authors of $[2,3]$ were able to reproduce the main effects linked to the "capacity drop" that are the Braess paradox and the "Faster Is Slower" effect, but not so much the self-organization. Our first goal is to further advance in this direction. We introduce a model which interpolates between two states of the traffic (organized and disorganized) which we represent by the presence of two levels of constraints and by an organization parameter which evolves through an ODE. This model admits a natural and efficient approximation strategy, relying on a combination of splitting, explicit Euler time integration and of a monotone finite volume scheme for LWR. In passing, we prove well-posedness for our model in Sections 1-2, but our main interest lies in the sections 3-4 where we perform a test to validate and discuss the model.

\section{Notion of solution and uniqueness}

Our starting point is the model (2) proposed by the authors of [4], see also [2, 3]. To go further, we introduce two levels of exit efficiencies $p_{\min } \leq p_{\max }$ (both are required to be Lipschitz continuous nonincreasing functions) and set

$$
q(t)=(1-\omega(t)) p_{\min }(\xi(t))+\omega(t) p_{\max }(\xi(t)), \quad \xi(t)=\int_{\mathbb{R}} \rho(x, t) \mu(x) \mathrm{d} x,
$$

where $\omega(t) \in(0,1)$ is an organization parameter which describes the state of the traffic and evolves through the ODE

$$
\dot{\omega}(t)=K(\xi(t), \dot{\xi}(t)) \omega(t)(1-\omega(t)) .
$$

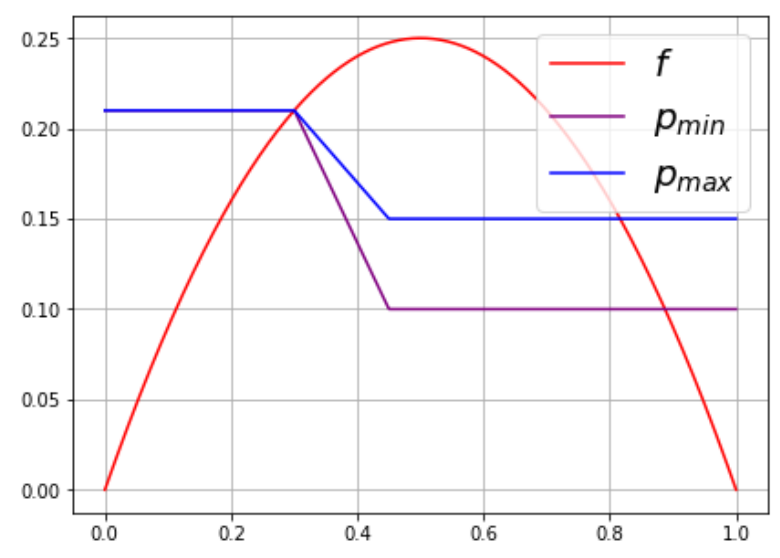

Figure 1: Typical behavior of exit efficiencies $p_{\min }, p_{\max }$ (left) and organization-driving function $K$ in (4) (right).

Mathematically speaking, we only suppose that $K \in \operatorname{Lip}_{\text {loc }}\left(\mathbb{R}^{2}\right)$. The idea behind phenomenologically relevant choices of $K$, see Figure 1(right), is to allow for progressive organization of traffic with time, while keeping the possibility of return to disorganization when sudden and strong variations of the traffic occur; see Section 4 . For the sake of being definite, in simulations we will choose $K$ under the form

$$
K(\xi, \chi)=C\left(\frac{\xi}{\xi_{c}}-1\right)^{+}\left(1-\frac{\chi^{+}}{D_{+}}-\frac{\chi^{-}}{D_{-}}\right),
$$

with some positive parameters $\xi_{c}, C, D_{+}, D_{-}$and the notations $z^{+}=\max \{z, 0\}, z^{-}=|z|-z^{+}$. This choice will be discussed later. To summarize, $q$ being given by (3)-(4), we have the following constrained PDE to study:

$$
\left\{\begin{array}{cc}
\partial_{t} \rho+\partial_{x}(f(\rho))=0 & \mathbb{R} \times(0, T) \\
\left.f(\rho)\right|_{x=0} \leq q(t) & t \in(0, T)
\end{array}\right.
$$


Frow now on, let us denote by $\Phi$ the entropy flux associated with the Kružkov entropy $\rho \mapsto|\rho-\kappa|$, for all $\kappa \in[0, R]$, see [23]. Following [16, 6, 14], we give the following definition of solution.

Definition 1.1. A couple $(\rho, \omega)$ with $\rho \in \mathbf{L}^{\infty}(\mathbb{R} \times(0, T)) \cap \mathbf{C}\left([0, T] ; \mathbf{L}_{\text {loc }}^{1}(\mathbb{R})\right)$ and $\boldsymbol{\omega} \in \mathbf{W}^{1, \infty}((0, T))$ is an admissible weak solution to the system $(3)-(6)$ with initial data $\left(\rho_{0}, \omega_{0}\right)$ if

(i) for all nonnegative test functions $\varphi \in \mathbf{C}_{c}^{\infty}(\mathbb{R} \times[0, T))$ and $\kappa \in[0, R]$, the following entropy inequalities are verified:

$$
\int_{0}^{T} \int_{\mathbb{R}}|\rho-\kappa| \partial_{t} \varphi+\Phi(\rho, \kappa) \partial_{x} \varphi \mathrm{d} x \mathrm{~d} t+\int_{\mathbb{R}}\left|\rho_{0}(x)-\kappa\right| \varphi(x, 0) \mathrm{d} x+2 \int_{0}^{T} \mathcal{R}(\kappa, q(t)) \varphi(0, t) \mathrm{d} t \geq 0,
$$

where $\mathcal{R}(\kappa, q(t))=f(\kappa)-\min \{f(\kappa), q(t)\}$;

(ii) for all nonnegative test functions $\psi \in \mathbf{C}_{c}^{\infty}((0, T))$ and some given $\varphi \in \mathbf{C}_{c}^{\infty}(\mathbb{R})$ which verifies $\varphi(0)=1$, the following weak constraint inequalities are verified:

$$
-\int_{0}^{T} \int_{\mathbb{R}^{+}} \rho \partial_{t}(\varphi \psi)+f(\rho) \partial_{x}(\varphi \psi) \mathrm{d} x \mathrm{~d} t \leq \int_{0}^{T} q(t) \psi(t) \mathrm{d} t
$$

(iii) the following weak ODE formulation is verified for all $t \in[0, T]$ :

$$
\omega(t)=\omega_{0}+\int_{0}^{t} K(\xi(s), \dot{\xi}(s)) \omega(s)(1-\omega(s)) \mathrm{d} s
$$

Remark 1.1. Let us underline that the formulation (7) - (9) above is stable with respect to the a.e. convergence of $\rho$.

Before we prove stability with respect to initial data and uniqueness for admissible weak solutions to the system (3) - (6), let us note that we can directly integrate the ODE (4). This feature is not crucial but it is practical.

Lemma 1.2. Fix $(\rho, \omega)$ an admissible weak solution to the system $(3)-(6)$. Then for all $t \in[0, T]$,

$$
\omega(t)=\frac{\exp (W(t))}{1+\exp (W(t))}, \quad W(t)=\ln \left(\frac{\omega_{0}}{1-\omega_{0}}\right)+\int_{0}^{t} K(\xi(s), \dot{\xi}(s)) \mathrm{d} s .
$$

Theorem 1.3. Suppose that $f$ satisfies (1). Fix $\rho_{0}^{1}, \rho_{0}^{2} \in \mathrm{L}^{1}(\mathbb{R} ;[0, R])$ and $\omega_{0}^{1}, \omega_{0}^{2} \in(0,1)$. We denote by $\left(\rho^{1}, \omega^{1}\right)$ and $\left(\rho^{2}, \omega^{2}\right)$ two admissible weak solutions to the system $(3)-(6)$ corresponding to the initial data $\left(\rho_{0}^{1}, \omega_{0}^{1}\right)$ and $\left(\rho_{0}^{2}, \omega_{0}^{2}\right)$, respectively. Then there exist constants $A, \alpha, \beta, \gamma$ such that if we note $G(z)=\exp \left(\beta z+\gamma z^{2} / 2\right)$, we have

$$
\text { for a.e. } t \in(0, T),\left\|\rho^{1}(t)-\rho^{2}(t)\right\|_{\mathrm{L}^{1}} \leq\left\|\rho_{0}^{1}-\rho_{0}^{2}\right\|_{\mathrm{L}^{1}} G(t)+\alpha\left|\mathrm{W}^{1}(0)-\mathrm{W}^{2}(0)\right| \int_{0}^{t} G(s) \mathrm{d} s
$$

and

$$
\forall t \in[0, T],\left|\omega^{1}(t)-\omega^{2}(t)\right| \leq\left(\frac{\left|\mathrm{W}^{1}(0)-\mathrm{W}^{2}(0)\right|}{4}\right)+A \int_{0}^{t}\left(\alpha\left|\mathrm{W}^{1}(0)-\mathrm{W}^{2}(0)\right|(t-s)+\left\|\rho_{0}^{1}-\rho_{0}^{2}\right\|_{\mathrm{L}^{1}}\right) G(s) \mathrm{d} s
$$

where $\mathrm{W}^{1}$ and $\mathrm{W}^{2}$ are defined as in Lemma 1.2. In particular, the system (3) - (6) admits at most one admissible weak solution.

Proof. First, a stability estimate [6, Prop. 2.10] characteristic of (2) yields Lipschitz continuous dependence $q \mapsto \rho$ for $q \in \mathbf{L}^{1}(0, T)$ and $\rho \in \mathbf{C}\left([0, T] ; \mathbf{L}^{1}(\mathbb{R})\right)$. Moreover, the map $\boldsymbol{\omega} \mapsto q$ for $\boldsymbol{\omega}, q \in \mathbf{C}([0, T])$ is obviously Lipschitz. Finally, by exploiting Lemma 1.2 and the fact that for a.e. $t \in(0, T), \dot{\xi}(t)=\int_{\mathbb{R}} f(\rho) \mu^{\prime}(x) \mathrm{d} x$, one can obtain Lipschitz dependence $\rho \mapsto \omega$, and Gronwall's lemma concludes. 


\section{Finite volume approximation of the model}

Here, we prove the existence of admissible weak solutions to the system (3) - (6). To do that, we construct and prove the convergence of an explicit Euler in time scheme for the ODE (4) combined with a monotone finite volume scheme for the constrained LWR (6). Fix $\rho_{0} \in \mathbf{L}^{1}(\mathbb{R} ;[0, R])$ and $\omega_{0} \in(0,1)$. For a fixed spatial mesh size $\Delta x$ and time mesh size $\Delta t$, let $x_{j}=j \Delta x, t^{n}=n \Delta t$. We define the grid cells and $N \in \mathbb{N}$ such that $T \in[N \Delta t,(N+1) \Delta t)$. We write

$$
\mathbb{R} \times[0, T] \subset \bigcup_{n=0}^{N} \bigcup_{j \in \mathbb{Z}} \mathcal{P}_{j+\frac{1}{2}}^{n}, \quad \mathcal{P}_{j+\frac{1}{2}}^{n}=\left(x_{j}, x_{j+1}\right) \times\left[t^{n}, t^{n+1}\right) .
$$

Denote by $\left(\rho_{j+\frac{1}{2}}^{0}\right)_{j \in \mathbb{Z}}$ and $\left(\mu_{j+\frac{1}{2}}\right)_{j \in \mathbb{Z}}$ suitable discretizations of the initial data $\rho_{0}$ and of the weight function $\mu$; for instance their mean values on each cell $\left(x_{j}, x_{j+1}\right)$.

\section{Initialization:}

$$
w^{0}=\omega_{0} \quad \text { and } \quad \xi^{0}=\sum_{j \in \mathbb{Z}} \rho_{j+\frac{1}{2}}^{0} \mu_{j+\frac{1}{2}} \Delta x
$$

Induction. Fix $n \in\{0, \ldots, N\}$.

At each time step, we first define a constraint level $q^{n}$ :

$$
q^{n}=\left(1-w^{n}\right) p_{\min }\left(\xi^{n}\right)+w^{n} p_{\max }\left(\xi^{n}\right) .
$$

We use this value to update the approximate traffic density with the marching formula

$$
\forall j \in \mathbb{Z}, \rho_{j+\frac{1}{2}}^{n+1}=\rho_{j+\frac{1}{2}}^{n}-\frac{\Delta t}{\Delta x}\left(\mathcal{F}_{j+1}^{n}\left(\rho_{j+\frac{1}{2}}^{n}, \rho_{j+\frac{3}{2}}^{n}\right)-\mathcal{F}_{j}^{n}\left(\rho_{j-\frac{1}{2}}^{n}, \rho_{j+\frac{1}{2}}^{n}\right)\right),
$$

where, given $\mathcal{F}=\mathcal{F}(x, y)$ a monotone and Lipschitz numerical flux consistent with $f$, following the recipe of [6, 14], we set

$$
\mathcal{F}_{j}^{n}(a, b)=\left\{\begin{array}{ccc}
\min \left\{\mathcal{F}(a, b), q^{n}\right\} & \text { if } & j=0 \\
\mathcal{F}(a, b) & \text { if } & j \neq 0 .
\end{array}\right.
$$

Then, setting $\xi^{n+1}=\sum_{j \in \mathbb{Z}} \rho_{j+\frac{1}{2}}^{n+1} \mu_{j+\frac{1}{2}} \Delta x$, we update the organization parameter

$$
\chi^{n+1}=\frac{\xi^{n+1}-\xi^{n}}{\Delta t}, \quad \theta^{n+1}=K\left(\xi^{n+1}, \chi^{n+1}\right) w^{n}\left(1-w^{n}\right), \quad w^{n+1}=w^{n}+\theta^{n+1} \Delta t .
$$

Conclusion. Define the functions

$$
\begin{aligned}
& \forall(x, t) \in \mathbb{R} \times(0, T), \rho^{\Delta}(x, t)=\sum_{n=0}^{N} \sum_{j \in \mathbb{Z}} \rho_{j+\frac{1}{2}}^{n} \mathbb{1}_{\mathcal{P}_{j+\frac{1}{2}}^{n}}(x, t) \\
& \forall t \in(0, T), q^{\Delta}(t), \chi^{\Delta}(t), \theta^{\Delta}(t)=\sum_{n=0}^{N}\left(q^{n}, \chi^{n+1}, \theta^{n+1}\right) \mathbb{1}_{\left[t^{n}, t^{n+1}\right)}(t) \\
& \forall t \in(0, T), \xi^{\Delta}(t)=\xi^{0}+\int_{0}^{t} \chi^{\Delta}(s) \mathrm{d} s, \quad \omega^{\Delta}(t)=w^{0}+\int_{0}^{t} \theta^{\Delta}(s) \mathrm{d} s .
\end{aligned}
$$

Let $\Delta=(\Delta x, \Delta t)$. For the convergence analysis, we will assume that $\Delta \rightarrow 0$, with $\lambda=\Delta t / \Delta x$ verifying the CFL condition

$$
\lambda \mathrm{L} \leq 1, \quad \mathrm{~L}=\left(\left\|\frac{\partial \mathcal{F}}{\partial x}\right\|_{\mathbf{L}^{\infty}}+\left\|\frac{\partial \mathcal{F}}{\partial y}\right\|_{\mathbf{L}^{\infty}}\right) .
$$




\subsection{Stability and discrete entropy inequalities}

Proposition 2.1 ( $\mathbf{L}^{\infty}$ stability). Given $q^{n}$ to define the constrained flux in (14), the scheme (13) is stable:

$$
\forall n \in\{0, \ldots, N\}, \forall j \in \mathbb{Z}, \rho_{j+\frac{1}{2}}^{n} \in[0, R] .
$$

Proof. One can follow the argumentation in [24, Proposition 3.1], or borrow elements from [20, Lemma 5.1] and [6, Proposition 4.2].

We now derive discrete entropy inequalities. These inequalities contain terms that will help to pass to the limit in the constrained formulation of the conservation law, as soon as the sequence $\left(q^{\Delta}\right)_{\Delta}$ of constraints is proved convergent as well. Let us note from now $a \vee b=\max \{a, b\}$ and $a \wedge b=\min \{a, b\}$.

Proposition 2.2 (Discrete entropy inequalities). The numerical scheme (12) - (15) fulfills the following inequalities for all $n \in\{0, \ldots, N-1\}, j \in \mathbb{Z}$ and $\mathrm{\kappa} \in[0, R]:$

$$
\left(\left|\rho_{j+\frac{1}{2}}^{n+1}-\kappa\right|-\left|\rho_{j+\frac{1}{2}}^{n}-\kappa\right|\right) \Delta x+\left(\Phi_{j+1}^{n}-\Phi_{j}^{n}\right) \Delta t \leq \mathcal{R}\left(\kappa, q^{n}\right) \Delta t \delta_{j \in\{-1,0\}}+\left(\Phi_{0}^{n}-\bar{\Phi}_{0}^{n}\right) \Delta t\left(\delta_{j=-1}-\delta_{j=0}\right),
$$

where

$$
\begin{aligned}
& \Phi_{j}^{n}=\mathcal{F}\left(\rho_{j-\frac{1}{2}}^{n} \vee \kappa, \rho_{j+\frac{1}{2}}^{n} \vee \kappa\right)-\mathcal{F}\left(\rho_{j-\frac{1}{2}}^{n} \wedge \kappa, \rho_{j+\frac{1}{2}}^{n} \wedge \kappa\right) \\
& \bar{\Phi}_{0}^{n}=\min \left\{\mathcal{F}\left(\rho_{-\frac{1}{2}}^{n} \vee \kappa, \rho_{\frac{1}{2}}^{n} \vee \kappa\right), q^{n}\right\}-\min \left\{\mathcal{F}\left(\rho_{-\frac{1}{2}}^{n} \wedge \kappa, \rho_{\frac{1}{2}}^{n} \wedge \kappa\right), q^{n}\right\} .
\end{aligned}
$$

Proof. This is a consequence of the scheme monotonicity. When the constraint does not enter the calculations i.e. $j \notin\{-1,0\}$, the proof follows [20, Lemma 5.4]. When the constraint enters the calculations, the constants $\mathrm{K} \in[0, R]$ are no longer stationary solutions of the scheme. Then, calculations make appear the term $\mathcal{R}\left(\kappa, q^{n}\right)$.

Starting from the marching formula (13) and the discrete entropy inequalities (18), we can derive approximate versions of (7) and (8). The proofs can be adapted from the ones of [12, Lemma 4.4] or [24, Proposition 3.3].

Proposition 2.3 (Approximate entropy/constraint inequalities). (i) Fix $\varphi \in \mathbf{C}_{c}^{\infty}\left(\mathbb{R} \times[0, T) ; \mathbb{R}^{+}\right), \mathrm{\kappa} \in[0, R]$. Then there exists a constant $\mathrm{C}_{1}^{\varphi}=\mathrm{C}_{1}^{\varphi}(R, T, \mathrm{~L})$, nondecreasing with respect to its arguments, such that

$$
\int_{0}^{T} \int_{\mathbb{R}}\left|\rho^{\Delta}-\kappa\right| \partial_{t} \varphi+\Phi^{\Delta}\left(\rho^{\Delta}, \kappa\right) \partial_{x} \varphi \mathrm{d} x \mathrm{~d} t+\int_{\mathbb{R}}\left|\rho_{0}^{\Delta}(x)-\kappa\right| \varphi(x, 0) \mathrm{d} x+2 \int_{0}^{T} \mathcal{R}\left(\kappa, q^{\Delta}(t)\right) \varphi(0, t) \mathrm{d} t \geq-\mathrm{C}_{1}^{\varphi}(\Delta t+\Delta x) .
$$

(ii) Fix $\psi \in \mathbf{C}_{c}^{\infty}\left((0, T) ; \mathbb{R}^{+}\right)$and $\varphi \in \mathbf{C}_{c}^{\infty}(\mathbb{R})$ such that $\varphi(0)=1$. Then there exists a constant $\mathrm{C}_{2}^{\varphi, \psi}=\mathrm{C}_{2}^{\varphi, \psi}\left(R, T, \mathrm{~L},\|f\|_{\mathrm{L}^{\infty}}\right)$, nondecreasing with respect to its arguments, such that

$$
-\int_{0}^{T} \int_{\mathbb{R}^{+}} \rho^{\Delta} \partial_{t}(\varphi \psi)+\mathcal{F}^{\Delta}\left(\rho^{\Delta}\right) \partial_{x}(\varphi \psi) \mathrm{d} x \mathrm{~d} t \leq \int_{0}^{T} q^{\Delta}(t) \psi(t) \mathrm{d} t+\mathrm{C}_{2}^{\varphi, \psi}(\Delta x+\Delta t),
$$

where

$$
\Phi^{\Delta}\left(\rho^{\Delta}, \kappa\right)=\sum_{n=0}^{N-1} \sum_{j \in \mathbb{Z}} \Phi_{j}^{n} \mathbb{1}_{\mathcal{P}_{j+\frac{1}{2}}^{n}}(x, t), \quad \mathcal{F}^{\Delta}\left(\rho^{\Delta}\right)=\sum_{n=0}^{N-1} \sum_{j \in \mathbb{Z}} \mathcal{F}\left(\rho_{j-\frac{1}{2}}^{n}, \rho_{j+\frac{1}{2}}^{n}\right) \mathbb{1}_{\mathcal{P}_{j+\frac{1}{2}}^{n}}(x, t) .
$$

The final step is to obtain compactness for the sequences $\left(\rho^{\Delta}\right)_{\Delta}$ and $\left(\omega^{\Delta}\right)_{\Delta}$ in order to pass to the limit in (19)-(20).

\subsection{Compactness and convergence}

Exploiting the compact embedding of $\mathbf{W}^{1, \infty}((0, T))$ in $\mathbf{C}([0, T])$, we can prove the existence of $\xi, \omega \in \mathbf{C}([0, T])$ such that (up to the extraction of a subsequence) $\left(\xi^{\Delta}\right)_{\Delta}$ and $\left(\omega^{\Delta}\right)_{\Delta}$ converge uniformly to $\xi$ and $\omega$, respectively. There are many ways to prove compactness of the sequence $\left(\rho^{\Delta}\right)_{\Delta}$. For example, one can derive weak BV estimates $([6,20])$ or use the singular mapping technique $([1,15])$. Here, since the conservation law in (6) is invariant under a translation in time, we derive local BV bounds, following [10, Lemma 4.2].

Proposition 2.4. Assume that $\rho_{0} \in \mathbf{B V}(\mathbb{R})$. Fix $0<\varepsilon<X$ and let $\Omega(\varepsilon, X)$ be the open subset $\Omega(\varepsilon, X)=(-X,-\varepsilon) \cup(\varepsilon, X)$. There exist two constants $\mathrm{C}_{3}$ and $\mathrm{C}_{4}>0$ such that for all $t \in[0, T-\Delta t)$,

$$
\operatorname{TV}\left(\rho^{\Delta}(t)_{\mid \Omega(\varepsilon, X)}\right) \leq \operatorname{TV}\left(\rho_{0}\right)+\frac{\mathrm{C}_{3}}{\varepsilon}, \quad \int_{\Omega(\varepsilon, X)}\left|\rho^{\Delta}(x, t+\Delta t)-\rho^{\Delta}(x, t)\right| \mathrm{d} x \leq \mathrm{C}_{4} \Delta t .
$$

Therefore, up to a subsequence, $\left(\rho^{\Delta}\right)_{\Delta}$ converges a.e. on $\mathbb{R} \times(0, T)$ to some $\rho \in \mathbf{L}^{\infty}(\mathbb{R} \times(0, T))$. 
Remark 2.1. At this point, the link between $\xi$ and $\rho$ is established: for a.e. $t \in(0, T), \xi(t)=\int_{\mathbb{R}} \rho(x, t) \mu(x) \mathrm{d} x$.

Theorem 2.5. Fix $\rho_{0} \in \mathbf{L}^{1}(\mathbb{R} ;[0, R]) \cap \mathbf{B V}(\mathbb{R})$ and $\omega_{0} \in(0,1)$. Suppose that $f$ satisfies (1) and that $\mu \in \mathbf{W}^{2,1}(\mathbb{R}) \cap \mathbf{C}^{2}(\mathbb{R})$. Then under the CFL condition (16), the scheme (12) - (15) converges to an admissible weak solution to the system (3) (6).

Proof. We show that the couple $(\rho, \omega)$ is a solution in the sense of Definition 1.1. First, let apply inequality (19) with $\varphi \in \mathbf{C}_{c}^{\infty}\left(\mathbb{R}^{*} \times[0, T) ; \mathbb{R}^{+}\right)$and $\kappa \in[0, R]$ to obtain

$$
\int_{0}^{T} \int_{\mathbb{R}}\left|\rho^{\Delta}-\kappa\right| \partial_{t} \varphi+\Phi^{\Delta}\left(\rho^{\Delta}, \kappa\right) \partial_{x} \varphi \mathrm{d} x \mathrm{~d} t+\int_{\mathbb{R}}\left|\rho_{0}^{\Delta}-\kappa\right| \varphi(x, 0) \mathrm{d} x \geq-\mathrm{C}_{1}^{\varphi}(\Delta x+\Delta t) .
$$

Then when letting $\Delta \rightarrow 0$, the a.e. convergence of $\left(\rho^{\Delta}\right)_{\Delta}$ to $\rho$ ensures that $\rho$ verifies $(7)$ away from the interface. Consequently, $\rho \in \mathbf{C}\left([0, T] ; \mathbf{L}_{\text {loc }}^{1}\left(\mathbb{R}^{*}\right)\right)$, see [11, Theorem 1.2]. Moreover, since $\rho$ is bounded and $\{x=0\}$ has a Lebesgue measure $0, \rho \in \mathbf{C}\left([0, T] ; \mathbf{L}_{\text {loc }}^{1}(\mathbb{R})\right)$. This ensures that the equality in Remark 2.1 actually holds for all $t \in[0, T]$. Moreover, since $\rho$ is an entropy solution in $\mathbb{R}^{*} \times(0, T)$ to $\partial_{t} \rho+\partial_{x}(f(\rho))=0$, $\xi$ defined in Remark 2.1 is actually in $\mathbf{W}^{1, \infty}((0, T))$ and verifies for a.e. $t \in(0, T)$,

$$
\dot{\xi}(t)=\int_{\mathbb{R}} f(\rho) \mu^{\prime}(x) \mathrm{d} x .
$$

We now pass to the limit in (19) and (20). The uniform convergence of both $\left(\xi^{\Delta}\right)_{\Delta}$ and $\left(\omega^{\Delta}\right)_{\Delta}$ ensures the existence of $q \in \mathbf{C}([0, T])$ such that $\left(q^{\Delta}\right)_{\Delta}$ converges to $q$ a.e. on $(0, T)$. Consequently, for a.e. $t \in(0, T)$,

$$
q(t)=(1-\omega(t)) p_{\min }(\xi(t))+\omega(t) p_{\max }(\xi(t)),
$$

and this equality actually holds for all $t \in[0, T]$ by continuity. Then by letting $\Delta \rightarrow 0$ in (19)-(20), we obtain that $(\rho, \omega)$ verifies the entropy inequalities (7) and the weak constraint inequalities (8).

An important step towards the assessment of the weak ODE formulation for $\omega$ is to show that $\left(\chi^{\Delta}\right)_{\Delta}$ converges a.e. to $\dot{\xi}$. One way to do that is by using a discrete integration by parts, assuming that $\mu \in \mathbf{W}^{2,1}(\mathbb{R}) \cap \mathbf{C}^{2}(\mathbb{R})$.

Corollary 2.6. Fix $\rho_{0} \in \mathbf{L}^{1}(\mathbb{R} ;[0, R]) \cap \mathbf{B V}(\mathbb{R})$ and $\omega_{0} \in(0,1)$. Suppose that $f$ satisfies $(1)$ and that $\mu \in \mathbf{W}^{2,1}(\mathbb{R}) \cap \mathbf{C}^{2}(\mathbb{R})$. Then the system (3) - (6) admits a unique admissible weak solution.

Proof. Uniqueness comes from Theorem 1.3, existence comes from Theorem 2.5, with a constructive proof.

Remark 2.2. Adopting the formalism proposed in [3], one could also prove well-posedness with fixed point arguments.

\section{Numerical simulations}

We report on numerical experiments with the scheme described in Section 2. We take the normalized uniformly concave flux $f(\rho)=\rho(1-\rho)$. We choose to use the Godunov flux at the interface $(j=0$ in (14)) and the Rusanov flux away from the interface $\left(j \neq 0\right.$ in (14)). A regularization of the function $\left.x \mapsto 2 n\left(x+\frac{1}{n}\right)\right) \mathbb{1}_{[-1 / n, 0]}(x)$ (with $\left.n=3\right)$ is issued as weight function. Following [3, Section 7], the setup for our simulation is as follows.

We consider the domain of computation $[-5,1]$, the initial data $\rho_{0}(x)=\mathbb{1}_{[-4,-2]}(x), \omega_{0}=0.2$ and the efficiencies of the exit $p_{\min }, p_{\max }$ are represented in Figure 1(left). For the simulations, we have fixed a locally Lipschitz prefactor $K$ in (4) with behaviour depicted in Figure 1 (right) and parameters $\xi_{c}=1 / 3, C=2 / 3, D_{+}=1 / 10$ and $D_{-}=D_{+} / 2$. The phenomenological features encoded in this choice will be addressed in Section 4.

We first address the error analysis in the above setup. Introduce the relative error $E^{\Delta}=\left\|\rho^{\Delta}-\rho^{\Delta / 2}\right\|_{\mathbf{L}^{1}\left((0, T) ; \mathbf{L}^{1}(\mathbb{R})\right)}$. In Table 1, we computed this error for different number of space cells at the final time $T=17$. We deduce that the order of convergence is approximately 0.852 . 


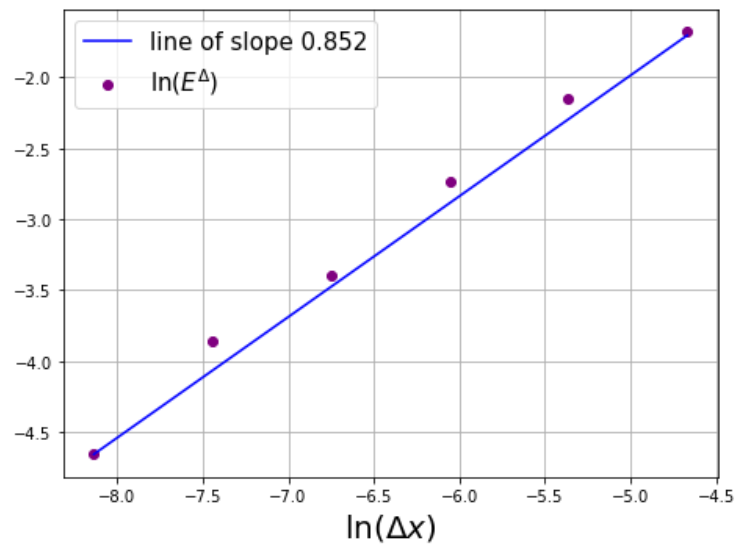

Figure 2: Rate of convergence.

Now, let us comment on qualitative features of the simulated traffic flow and provide its interpretation in terms of agents' behaviors. First, as we can see in Figure 3, the introduction of the organization parameter favors the evacuation time.
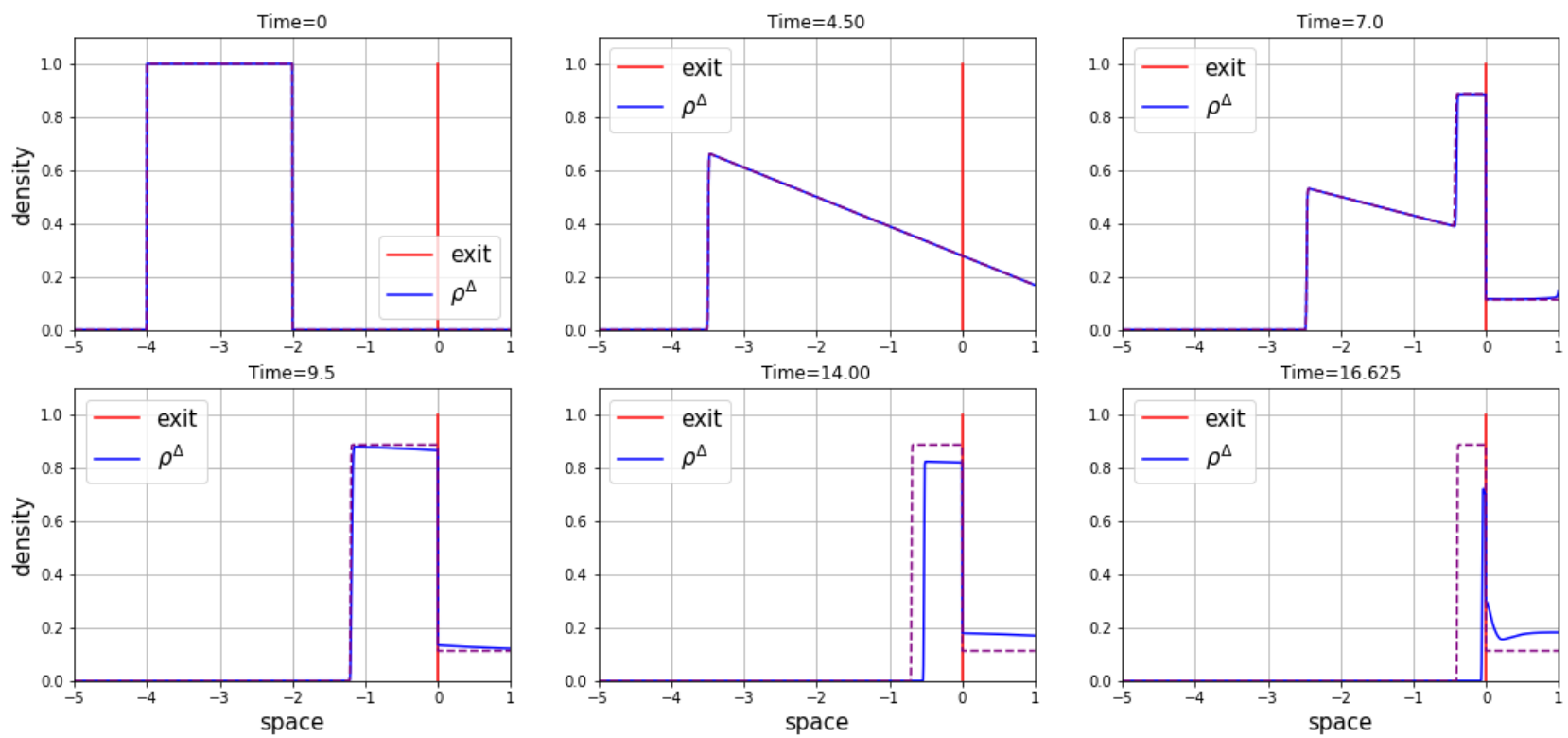

Figure 3: The numerically computed solution $x \mapsto \rho^{\Delta}(x, t)$ at different fixed times $t$; dashed lines correspond to the reference solution in absence of self-organization $\omega=0$ in (3).

Figure 4 highlights the fact that the model reproduces some features expected from self-organization. At first, the exit flux increases until it reaches the maximum level of the exit efficiency. As traffic densifies, the exit flux falls down to the lowest value of this efficiency, which reflects rapid disorganization, i.e., predominance of agents' individualistic strategies over the rational collective behavior. Then, in the time interval $[6,16]$, the elevated density upstream has very small variations which leads to the emergence of a coherent collective behavior of the agents. This is witnessed through the increase of both the organization marker and the exit flux. We stress out that without self-organization, the exit flux keeps its minimal value in this time interval. Then a notable phenomenon seems to take place. In the time interval $[15.5,16.3]$, the jam upstream the exit starts to resorb, and the exit efficiency (which is monitored by the exit flux) slightly falls down while the organization level regresses significantly. In other words, the agents abandon collective strategies in rapidly evolving environments, but this does not affect the traffic dramatically because densities are also strongly decreased. 

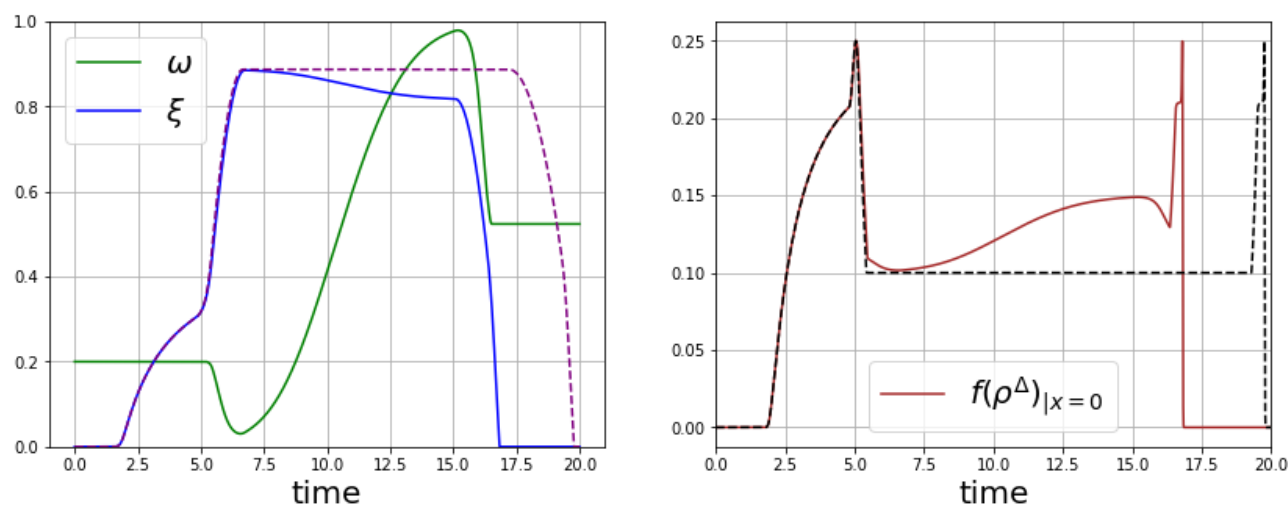

Figure 4: Left: subjective density $\xi$ and organization marker $\omega$. Right: exit flux $\left.f(\rho)\right|_{x=0^{-}}$; dashed lines correspond to the reference solution in absence of self-organization $\omega=0$ in (3).

\section{Conclusions and perspectives}

The model we propose here permits a rigorous analysis of well-posedness as well as a robust and simple numerical approximation. It enriches the qualitative behavior of the simple LWR-based models for bottlenecks ([16, 4, 3]), due to its ability to reproduce a few self-organization features. Let us deeper discuss the model construction, in particular the role of the function $K$ whose behavior is depicted in Figure 1(right). Its key features are as follows:

- invariance of the organization marker $\omega$ in the region of low densities;

- rapid decrease of $\omega$ for moderate and particularly for high densities, under strong density variations;

- progressive increase of $\omega$ in dense and very dense traffic with small density variations.

The idea behind these features is: rapidly changing traffic conditions, at considerable densities, promote individual behavior and rapidly lead to a somewhat chaotic interactions among agents, thus lowering the exit efficiency; while persistent coercive traffic conditions, such as a jam, help to emerge and promote a collective behavior like formation of well-organized queues, the alternate in the order of passage through the bottleneck, and a higher degree of mutual courtesy among agents; thus the exit efficiency improves accordingly, which enhances the jam evacuation. The form (5) provides a simple example of such behavior, which is confirmed by the simulations of Section 3. The parameter $\xi_{c}$ has the meaning of activation threshold for organization/disorganization of the traffic at bottleneck; $D_{+}, D_{-}$indicate thresholds of transition from cooperative (low variations of $\xi$ ) to individualistic (higher ones) dynamics of agents.

One way to improve this model would be to take into account unexpected/rash behavior of certain agents. Let us recall that unlike fluid mechanics models, traffic models deal with a relatively small number of agents. In consequence, we would expect the dynamics to be greatly impacted by the behavior of a few agents. An idea to model such rash behaviors is to introduce a stochastic term in the definition of the prefactor $K$, for example

$$
K(t, \xi, \chi)=C\left(\frac{\xi}{\xi_{c}}-1\right)^{+}\left(1-\frac{\chi^{+}}{D_{+}}-\frac{\chi^{-}}{D_{-}}-X(t)\right)
$$

where $X$ is a stochastic process modeling the harmful impact of a random number of mindless agents on the collective dynamics. We plan to study numerically this variant of the model and provide indications concerning the impact of undisciplined agents on the evacuation time.

In the forthcoming work [7], we will take inspiration from second-order macroscopic models of traffic [8, 25] to model self-organization globally on the road; note that bottlenecks can be as well modelled with non-local point constraints within such models, see, e.g., [5]. Mimicking the key elements (3)-(4) of the model we addressed in the present note, we will introduce two fundamental graphs $f_{\min } \leq f_{\max }$ to describe the two states of the traffic and make the space-and-time dependent organization parameter act both on the constraint levels (3) and on the fundamental graphs. We will then have to study a variant of nonlocal LWR model, cf. [9,21] for related mathematical and numerical issues. 


\section{References}

[1] Adimurthi, J. Jaffré, and G. D. V. Gowda. Godunov-type methods for conservation laws with a flux function discontinuous in space. SIAM J. Numer. Anal., 42(1):179-208, 2005.

[2] B. Andreianov, C. Donadello, U. Razafison, and M. D. Rosini. Qualitative behaviour and numerical approximation of solutions to conservation laws with non-local point constraints on the flux and modeling of crowd dynamics at the bottlenecks. ESAIM: M2AN, 50(5):1269-1287, 2016.

[3] B. Andreianov, C. Donadello, U. Razafison, and M. D. Rosini. Analysis and approximation of one-dimensional scalar conservation laws with general point constraints on the flux. J. Math. Pures et Appl., 116:309-346, 2018.

[4] B. Andreianov, C. Donadello, and M. D. Rosini. Crowd dynamics and conservation laws with nonlocal constraints and capacity drop. Math. Models Methods in Appl., 24(13):2685-2722, 2014.

[5] B. Andreianov, C. Donadello, and M. D. Rosini. A second-order model for vehicular traffics with local point constraints on the flow. Math. Models Methods in Appl., 26(4):751-802, 2016.

[6] B. Andreianov, P. Goatin, and N. Seguin. Finite volume schemes for locally constrained conservation laws. Numer. Math., 115(4):609-645, 2010.

[7] B. Andreianov and A. Sylla. An hybrid LWR model to reproduce self-organization of traffic. In preparation.

[8] A. Aw and M. Rascle. Resurrection of "second order" models of traffic flow. SIAM J. Appl. Math, 60(3):916-938, 2000.

[9] S. Blandin and P. Goatin. Well-posedness of a conservation law with non-local flux arising in traffic flow modeling. Numer. Math., 132(2):217-241, 2016.

[10] R. Bürger, A. García, K. H. Karlsen, and J. D. Towers. A family of numerical schemes for kinematic flows with discontinuous flux. J. Engrg. Math., 60:387-425, 2008.

[11] C. Cancès and T. Gallouët. On the time continuity of entropy solutions. J. Evol. Equ., 11(1):43-55, 2011.

[12] C. Cancès and N. Seguin. Error estimate for godunov approximation of locally constrained conservation laws. SIAM J. Numer. Anal., 50(6):3036-3060, 2012.

[13] E. M. Cepolina. Phased evacuation: An optimisation model which takes into account the capacity drop phenomenon in pedestrian flows. Fire Saf. J., 44(4):532-544, 2009.

[14] C. Chalons, P. Goatin, and N. Seguin. General constrained conservation laws. application to pedestrian flow modeling. Networks Heterogen. Media, 2(8):433-463, 2013.

[15] G. Coclite and H. Risebro. Conservation laws with time dependent discontinuous coefficients. SIAM J. Math. Analysis, 36:1293-1309, 2005.

[16] R. M. Colombo and P. Goatin. A well posed conservation law with a variable unilateral constraint. J. Differ. Equ., 234(2):654$675,2007$.

[17] R. M. Colombo and M. Rosini. Pedestrian flows and non-classical shocks. Math. Methods Appl. Sci., 28(28):1553-1567, 2005.

[18] E. Cristiani, B. Piccoli, and A. Tosin. How can macroscopic models reveal self-organization in traffic flow? In 2012 IEEE 51st IEEE Conference on Decision and Control (CDC), pages 6989-6994, 2012.

[19] M. L. Delle Monache and P. Goatin. Scalar conservation laws with moving constraints arising in traffic flow modeling: an existence result. J. Differ. Equ., 257(11):4015--4029, 2014.

[20] R. Eymard, T. Gallouët, and R. Herbin. Finite Volume Methods, volume VII of Handbook of Numerical Analysis. North-Holland, Amsterdam, 2000.

[21] P. Goatin and S. Scialanga. Well-posedness and finite volume approximations of the lwr traffic flow model with non-local velocity. Networks Heterogen. Media, 11(1):107-121, 2016.

[22] B. S. Kerner. Experimental features of self-organization in traffic flow. Phys. Rev. Lett., 81:3797-3800, 1998.

[23] S. N. Kružhkov. First order quasilinear equations with several independent variables. Mat. Sb. (N.S.), 81(123):228-255, 1970.

[24] A. Sylla. Influence of a slow moving vehicle on traffic: Well-posedness for a mildly nonlocal model. https://hal.archivesouvertes.fr/hal-02391844, Dec 2019.

[25] H. M. Zhang. A non-equilibrium traffic model devoid of gas-like behavior. Transport. Res. Part B, 36(3):275-290, 2002. 